\title{
Síndrome de piernas inquietas en pacientes con concentración de ferritina elevada y hierro normal
}

\author{
Rosa Peraita-Adrados, Luis G. Duque-Ramírez, Antonio Vela-Bueno
}

Objetivo. Documentar la asociación entre síndrome de piernas inquietas (SPI) y concentraciones de ferritina elevadas en cinco pacientes.

Pacientes y métodos. Estudiamos a cinco varones con una edad media de 59 años (rango: 36-73 años). Todos los pacientes fueron remitidos por SPI (dos de ellos donantes de sangre), en dos casos asociado a síndrome de apnea obstructiva del sueño. Se efectuaron registros videopolisomnográficos y se realizó una analítica para determinar los niveles de ferritina y hierro en plasma.

Resultados. Los cinco pacientes presentaban criterios clínicos de SPI: parestesias en las pantorrillas asociadas a una necesidad imperiosa de mover las piernas, inquietud motora, agravamiento de los síntomas por la tarde y por la noche, mejoría con el movimiento, dificultad de conciliación del sueño y despertares nocturnos frecuentes. La exploración neurológica, el electroencefalograma, el electromiograma y la resonancia magnética cerebral fueron normales. Los registros videopolisomnográficos mostraron sueño nocturno fragmentado, reducción del tiempo total de sueño, escasa eficiencia, índice de apnea-hipopnea $>10 / \mathrm{h}$ en dos casos, y en los cinco casos, índice de movimientos periódicos de las piernas por hora de sueño $>5 / \mathrm{h}$. En todos los casos los niveles de hierro sérico estaban dentro de los límites normales y la concentración de ferritina era elevada.

Conclusiones. La asociación entre SPI con movimientos periódicos de las piernas durante el sueño, hierro sérico normal y ferritina elevada no se ha descrito previamente. El hallazgo de la disminución de concentración de ferritina en uno de los pacientes -meses más tarde del tratamiento con fármacos dopaminérgicos-apoya la implicación de un mecanismo dopaminérgico en la fisiopatología del SPI.

Palabras clave. Ferritina elevada. Hierro normal. Movimientos periódicos de las piernas. Síndrome de piernas inquietas. Videopolisomnografía.

\section{Introducción}

En 1945 Ekbom reconoce como una enfermedad distinta el síndrome de piernas inquietas (SPI) y lo denomina restless legs syndrome, enfermedad que se había descrito tres siglos antes [1,2]. Otros autores habían señalado que además de las sensaciones disestésicas profundas y mal definidas en las pantorrillas, que aparecen en reposo e inducen a mover las piernas con carácter imperioso, los pacientes presentaban sacudidas en las piernas [3-7].

El diagnóstico es clínico; se basa en la historia clínica, las escalas de gravedad y el Suggested Immobilization Test [6-8]. El paciente suele consultar en la unidad de sueño por un insomnio debido a dificultad para conciliar o mantener el sueño, o por somnolencia diurna. El registro polisomnográfico (PSG) es obligatorio cuando se sospecha comorbilidad con otra patología del sueño - por ejemplo, síndrome de apnea/hipopnea obstructiva del sueño (SAHOS) - y el tratamiento va dirigido a la correc- ción de ambas patologías. La prevalencia del SPI se estima en torno al 2-3\% en adultos $[9,10]$. Existen formas primarias, a menudo con un patrón familiar, y formas sintomáticas o secundarias por déficit de hierro, embarazo, uremia, diabetes y neuropatía. Entre las formas secundarias, la ferropenia (ferritina plasmática inferior a $50 \mu \mathrm{g} / \mathrm{L}$ ) es la primera causa que se debe tener en cuenta debido a que en la fisiopatología del SPI el metabolismo del hierro en el cerebro es anormal, con una disminución del hierro intracelular y una alteración del sistema dopaminérgico central. El hierro, como se sabe, participa en el metabolismo de la dopamina y es necesario para la transformación de tirosina en dopamina [11, 12]. La disminución de las concentraciones plasmáticas de ferritina influye en la presencia o gravedad del síndrome. Es necesario realizar a los pacientes con SPI un estudio completo del metabolismo del hierro, incluyendo determinación de hierro sérico, ferritina, transferrina e índice de saturación o receptores solubles de transferrina.
Unidad de Sueño y Epilepsia; Servicio de Neurofisiología Clínica; Hospital Universitario Gregorio Marañón (R. Peraita-Adrados, L.G. Duque-Ramírez). Laboratorio de Sueño Humano y Cronobiología Aplicada; Universidad Autónoma de Madrid. Madrid, España (L.G. Duque-Ramírez, A. Vela-Bueno).

Correspondencia:

Dra. Rosa Peraita Adrados. Unidad de Sueño y Epilepsia. Servicio de Neurofisiología Clínica. Hospital General Universitario Gregorio Marañón. Doctor Esquerdo, 46. E-28007 Madrid.

Fax: +34 915868018

E-mail:

mperaita.hgugm@ salud.madrid.org

Financiación:

L.G.D.R. es becario de la Universidad de Antioquia, Facultad de Medicina, Departamento de Fisiología y Bioquímica (Medellín, Colombia).

Trabajo presentado como póster en el 18. Congreso de la European Sleep Research Society, celebrado en Innsbruck (Austria) en 2006.

Aceptado tras revisión externa: 11.05.11.

Cómo citar este artículo: Peraita-Adrados R, Duque-Ramírez LG, Vela-Bueno A. Síndrome de piernas inquietas en pacientes con concentración de ferritina elevada y hierro normal. Rev Neurol 2011; 53: 385-9.

(c) 2011 Revista de Neurología 
Tabla I. Edad, diagnóstico de referencia, antecedentes patológicos, escala de somnolencia de Epworth e índice de masa corporal de los pacientes.

\begin{tabular}{lcccccc}
\hline & Sexo & $\begin{array}{c}\text { Edad } \\
\text { (años) }\end{array}$ & ESS & $\begin{array}{c}\text { IMC } \\
\left(\mathrm{kg} / \mathrm{m}^{2}\right)\end{array}$ & $\begin{array}{c}\text { Diagnóstico } \\
\text { de referencia }\end{array}$ & $\begin{array}{c}\text { Otros } \\
\text { diagnósticos }\end{array}$ \\
\hline Caso 1 & Varón & 61 & 9 & 30,7 & SPI & SAHOS, donante \\
\hline Caso 2 & Varón & 73 & 9 & 31,8 & SPI & IRC, nefrectomía, diálisis \\
\hline Caso 3 & Varón & 56 & 16 & 25,4 & SPI & IAM, HTA, dislipidemia, gota \\
\hline Caso 4 & Varón & 36 & - & 25,0 & SPI & Dislipidemia, alcohol \\
\hline Caso 5 & Varón & 48 & 16 & 30,6 & SPI & SAHOS, donante \\
\hline
\end{tabular}

ESS: escala de somnolencia de Epworth; HTA: hipertensión arterial; IAM: infarto agudo de miocardio; IMC: índice de masa corporal; IRC: insuficiencia renal crónica, SAHOS: síndrome de apnea/hipopnea obstructiva del sueño; SPI: síndrome de piernas inquietas.

El objetivo del trabajo es describir varios casos de pacientes con concentraciones de ferritina elevada que paradójicamente presentaban un SPI, observación que no se ha publicado previamente.

\section{Pacientes y métodos}

Estudiamos a cinco pacientes varones con una edad media de 59 años (rango: 36-73 años), remitidos a la unidad de sueño con el diagnóstico de SPI. Uno de ellos, con insuficiencia renal crónica de origen tuberculoso en tratamiento con hemodiálisis; otro, con antecedentes de SPI, alcoholismo (transaminasas y gamma glutamil transpeptidasa normales) y dislipidemia, y un tercero, con antecedentes de hipertensión arterial e infarto agudo de miocardio. Dos pacientes tenían además un diagnóstico de SAHOS (uno de ellos con diabetes mellitus tipo 2, hipotiroidismo subclínico e historia de donante de sangre durante 15 años) y otro también donante de sangre.

La tabla I muestra el diagnóstico de referencia y los antecedentes patológicos de los pacientes. Se tuvieron en cuenta para el diagnóstico de SPI los criterios clínicos de Walters et al [13] y el cuestionario específico del Johns Hopkins [14] traducido al castellano con autorización del Dr. Allen y realizado, en vez de por teléfono, de forma presencial en consulta de la unidad. Para la medida de la somnolencia diurna se utilizó la escala de somnolencia de Epworth (ESS) [15]. La exploración física (antropometría e índice de masa corporal) se completó con el examen neurológico, el electroencefalograma (EEG) de vigilia, el electromiograma (EMG) y la renosancia magnética (RM) cerebral.
Se realizó al menos un registro video-PSG nocturno: cinco canales de EEG, dos canales de oculografía, electrocardiograma, EMG de los músculos submentonianos y de ambos músculos tibiales anteriores, flujo aéreo nasobucal (mediante cánula), esfuerzo torácico-abdominal y saturación de oxígeno. El análisis del PSG se llevó a cabo siguiendo los criterios establecidos en el manual de Rechtschaffen y Kales [16] en períodos de 30 segundos.

La analítica consistió en bioquímica, hemograma, hierro, ferritina, transferrina, índice de saturación de la transferrina o receptores de transferrina.

\section{Resultados}

Todos los pacientes reunían criterios clínicos de SPI. En la exploración física destacaba un sobrepeso en cuatro de ellos con un índice de masa corporal medio de $28,04 \mathrm{~kg} / \mathrm{m}^{2}$ (rango: $25-31,8 \mathrm{~kg} / \mathrm{m}^{2}$ ) (Tabla I). La ESS mostró una puntuación media de 12,5 en cuatro pacientes, lo que refleja una somnolencia diurna moderada. El examen neurológico, el EEG, el EMG (en cuatro pacientes) y la RM cerebral fueron normales. Los registros PSG mostraron un sueño nocturno perturbado y fragmentado con una disminución del tiempo total de sueño y del índice de eficiencia del sueño (IES), un aumento del tiempo de vigilia después del comienzo del sueño y del número de eventos respiratorios obstructivos en dos pacientes (índice de apnea-hipopnea por hora de sueño $>10 / \mathrm{h}$ ) y, en todos los pacientes, un índice de movimientos periódicos en las piernas clínicamente significativo: > 5/h (Tabla II). En el caso 2, el que presentaba el SPI más grave asociado a SAHOS, 
Tabla II. Análisis paramétrico del polisomnograma.

\begin{tabular}{lcccccccccc}
\hline & TTS & WASO & LS & LREM & $\%$ no REM & $\%$ REM & MPP/h & IES & IF & IAH \\
\hline Caso 1 & 375 & 54 & 4 & 65 & 93,8 & 6,3 & 9,2 & 86,4 & 30 & 30 \\
\hline Caso 2 & 171 & 277 & 29 & - & 100,0 & 0 & 59,6 & 37,3 & 53,9 & 34,4 \\
\hline Caso 3 & 335 & 105 & 14 & 127,5 & 91,4 & 8,6 & 45,5 & 72 & 28,8 & 9 \\
\hline Caso 4 & 311 & 49 & 91 & 72 & 81,0 & 19,0 & 23,5 & 60 & 30,3 & 0 \\
\hline Caso 5 & 286 & 135 & 11 & 229 & 81,8 & 18,15 & 25,3 & 65,1 & 19 & 0 \\
\hline
\end{tabular}

IAH: índice de apnea-hipopnea por hora de sueño; IES: índice de eficiencia del sueño (tiempo dormido/tiempo en cama); IF: índice de fragmentación del sueño (número de cambios de fase/hora de sueño); LS: latencia del sueño; LREM: latencia de la primera fase rapid eye movement; MPP/h: índice de movimientos de las piernas por hora de sueño; TTS: tiempo total de sueño; WASO: tiempo de vigilia después del comienzo del sueño.

Tabla III. Analítica: hemograma.

\begin{tabular}{|c|c|c|c|c|c|c|}
\hline Caso 2 & 4,34 & 11,4 & 35,7 & 82,2 & 26,3 & 31,9 \\
\hline Caso 4 & 4,55 & 14,8 & 41,7 & 91,6 & 32,5 & 35,5 \\
\hline Caso 5 & 5,53 & 16,7 & 48,3 & 87,4 & 30,2 & 34,6 \\
\hline
\end{tabular}

Entre paréntesis, valores normales. HCM: hemoglobina corpuscular media; VCM: volumen corpuscular medio.

no se registró fase REM (rapid eye movement) en toda la noche y el IES fue tan sólo del $37 \%$. El hemograma mostró en este mismo paciente una anemia con una hemoglobina de 11,4 debido a su insuficiencia renal crónica en tratamiento con hemodiálisis (Tabla III). Este paciente estaba tratado con eritropoyetina (4.000 U subcutáneas tras cada diálisis) y hierro oral.

Los niveles de hierro sérico estaban dentro de los límites normales $(64-150 \mu \mathrm{g} / \mathrm{dL})$ en los cinco pacientes, mientras que los niveles de ferritina estaban aumentados en todos ellos (350-735,9 $\mu \mathrm{g} / \mathrm{dL})$, por encima del rango máximo de los valores de referencia (Tabla IV).

Con respecto al tratamiento, inicialmente se trató a un paciente con pramipexol, a tres con clonacepam y a otro con levodopa. Después del estudio completo en la unidad de sueño todos los pacientes fueron tratados con pramipexol, y en los dos pa- cientes con SAHOS se prescribió presión aérea positiva continua nasal domiciliaria.

El caso 2 falleció a los tres años de tratamiento como consecuencia de un hematoma subdural tras un traumatismo craneoencefálico por una caída accidental.

\section{Discusión}

La principal causa secundaria del SPI es la ferropenia, y su evaluación es primordial en todo paciente con sintomatología de SPI. El grupo del Johns Hopkins [17] ha evaluado el estado del hierro de los pacientes con SPI a través de varios métodos: concentración de ferritina en plasma o en el líquido cefalorraquídeo, RM cerebral y estudio post mortem del tejido nervioso. Estas determinaciones demuestran que hay una deficiencia de hierro en el cerebro en 
Tabla IV. Hierro y proteínas.

\begin{tabular}{|c|c|c|c|c|c|}
\hline & $\begin{array}{c}\text { Hierro } \\
\text { (59-158 } \mu \mathrm{g} / \mathrm{dL})\end{array}$ & $\begin{array}{c}\text { Índice de saturación } \\
\text { de transferrina } \\
(20-40 \%)\end{array}$ & $\begin{array}{c}\text { Transferrina } \\
(200-360 \mathrm{mg} / \mathrm{dL})\end{array}$ & $\begin{array}{c}\text { Receptores } \\
\text { de transferrina } \\
(0,83-1,76 \mathrm{mg} / \mathrm{L})\end{array}$ & $\begin{array}{c}\text { Ferritina } \\
(26-350 \mu \mathrm{g} / \mathrm{L})\end{array}$ \\
\hline Caso 1 & 150 & - & 197 & 1,49 & 350 \\
\hline Caso 2 & 68 & 60 & 140 & - & 438 \\
\hline Caso 3 & 75 & 30 & 200 & - & 437 \\
\hline Caso 4 & 117 & - & 308 & - & 735,9 \\
\hline Caso 5 & 64 & - & 221 & 1,39 & 380 \\
\hline
\end{tabular}

Entre paréntesis, valores normales.

pacientes con SPI en relación con controles normales. Paradójicamente, el receptor de transferrina aparece disminuido [18] cuando debería estar aumentado. La regulación del receptor de transferrina en pacientes con SPI podría estar alterada, de manera que afectaría al sistema que suministra niveles de hierro normales para el funcionamiento celular en el sistema nervioso central.

En nuestros pacientes con SPI hemos realizado la analítica habitual y hallamos concentraciones plasmáticas normales de hierro, bajas de transferrina y elevadas de ferritina.

Para O'Keeffe [19] la ferropenia se observa en el $22 \%$ de los pacientes con comienzo de la sintomatología en torno a los 50 años, y este porcentaje crece a medida que aumenta la edad: un $58 \%$ a los 64 años $(p=0,009)$. También la neuropatía es más frecuente en el SPI de comienzo tardío $(p=0,08)$. Según este autor los antecedentes familiares son menos habituales a medida que la edad de comienzo de los síntomas es más tardía [20].

En nuestra serie, sólo un paciente de 36 años (caso 4) presentaba antecedentes familiares de SPI.

Los niveles de ferritina son equívocos en determinadas circunstancias, como la coexistencia de un proceso inflamatorio. En estas condiciones, cuando los niveles de ferritina son paradójicos (deficiencia de hierro y nivel de ferritina normal), se debe analizar la medula ósea [21]. Ninguno de nuestros pacientes presentaba en el momento del diagnóstico de SPI comorbilidad con una enfermedad inflamatoria y en ningún caso nos planteamos un estudio de la medula ósea.

En el trabajo de Haba-Rubio et al [22], en dos pacientes con hemocromatosis y SPI estudiados con
RM cerebral y secuencias R2', comparados con nueve controles sanos, se observó una deficiencia local de hierro en la sustancia negra, el núcleo rojo y el globo pálido en los pacientes, lo que sugiere un papel del metabolismo del hierro en la fisiopatología del SPI. En nuestros pacientes no se ha realizado un estudio de RM cerebral de estas características, por lo que desconocemos la deficiencia o no de hierro en el cerebro; ahora bien, sería plausible que nuestros casos pudieran tratarse de formas de hemocromatosis subclínicas y que tuvieran depleción de hierro en el sistema nervioso central como los casos de Haba-Rubio et al.

La observación de pacientes donantes de sangre con SPI es relativamente frecuente en nuestro medio. Estos pacientes (más en mujeres) suelen tener unos niveles de ferritina inferiores a $50 \mu \mathrm{g} / \mathrm{L}$. La donación de una unidad de sangre produce una pérdida aproximada de $236 \mathrm{mg}$ de hierro, que requiere 3-4 meses para recuperarse con una dieta normal. En el sexo masculino las donaciones permitidas son cuatro al año, con dos meses de intervalo entre ellas, y curiosamente no se determina la ferritina previamente. Resulta por tanto infrecuente y de difícil interpretación que dos pacientes de nuestra serie -donantes de sangre durante más de 10 añospresentaran unos niveles de ferritina elevados.

El hallazgo de la disminución de concentración plasmática de ferritina -meses más tarde del tratamiento con fármacos dopaminérgicos- en uno de los pacientes (caso 4) sugiere la implicación de un mecanismo dopaminérgico en la fisiopatología del SPI -consideración especulativa, pero que planteamos ante la posibilidad de otros estudios con casos análogos-. 
Bibliografía

1. Ekbom KA. Restless legs. Acta Med Scand 1945; Suppl 158: $1-123$.

2. Willis T. De animae brutorum. London: Wells \& Scott; 1672.

3. Lugaresi E, Coccagna G, Tassinari CA, Ambrosetto C. Particularités cliniques et polygraphiques du syndrome d'impatience des membres inférieurs. Rev Neurol (Paris) $1965 ; 113: 545$

4. Coccagna G, Lugaresi E. Insomnia in the restless legs syndrome. In Gastaut H, Lugaresi E, Berti Ceroni G, Coccagna G, eds. The abnormalities of sleep in man. Bologna: A. Gaggi Editore; 1968. p. 285-94.

5. Lugaresi E, Cirignotta F, Coccagna G, Montagna P. Nocturnal myoclonus and restless legs syndrome. Adv Neurol 1986; 43: 295-307.

6. Michaud M, Lavigne G, Desautels A, Poirier G, Montplaisir J. Effects of immobility on sensory and motor symptoms of restless legs syndrome. Mov Disord 2002; 17: 112-5.

7. Michaud M, Paquet J, Lavigne G, Desautels A, Montplaisir J. Sleep laboratory diagnosis of restless legs syndrome. Eur Neurol 2002; 48: 108-13.

8. Walters AS, International Restless Legs Syndrome Study Group. Toward a better definition of the restless legs syndrome. Mov Disord 1995; 10: 634-42.

9. Phillips B, Young T, Finn L, Asher K, Hening WA, Purvis C. Epidemiology of restless legs symptoms in adults. Arch Intern Med 2000; 160: 2137-41.

10. Allen RP, Walters AS, Montplaisir J, Hening W, Myers A, Bell TJ, et al. Restless legs syndrome prevalence and impact: REST general population study. Arch Intern Med 2005; 165 : 1286-92.

11. Allen R. Dopamine and iron in the pathophysiology of restless legs syndrome (RLS). Sleep Med 2004; 5: 385-91.

12. Wang X, Wiesinger J, Beard J, Felt B, Menzies S, Earley C, et al. Thy 1 expression in the brain is affected by iron and is decreased in restless legs syndrome. J Neurol Sci 2004; 220: 59-66.

13. Walters AS, LeBrocq C, Dhar A, Hening W, Rosen R, Allen $\mathrm{RP}$, et al. Validation of the International Restless Legs Syndrome Study Group rating scale for restless legs syndrome. Sleep Med 2003; 4: 121-32.

14. Hening WA, Allen RP, Thanner S, Washburn T, Heckler D, Walters AS, et al. The Johns Hopkins telephone diagnostic interview for the restless legs syndrome: preliminary investigation for validation in a multi-center patient and control population. Sleep Med 2003; 4: 137-41.

15. Johns MW. A new method for measuring daytime sleepiness: the Epworth Sleepiness Scale. Sleep 1991; 14: 540-5.

16. Rechtschaffen A, Kales A. A manual of standardized terminology, techniques and scoring system for sleep stages in human subjects. Public Health Service Publication n. 204. Washington DC: US Government Printing Office; 1968.

17. Earley CJ, B Barker P, Horská A, Allen RP. MRI-determined regional brain iron concentrations in early- and late-onset restless legs syndrome. Sleep Med 2006; 7: 458-61.

18. Connor JR, Boyer PJ, Menzies SL, Dellinger B, Allen RP, Ondo WG, et al. Neuropathological examinations suggests impaired brain iron acquisition in restless legs syndrome. Neurology 2003; 61: 304-9.

19. O'Keeffe ST, Gavin K, Lavan JN. Iron status and restless legs syndrome in the elderly. Age Ageing 1994; 23: 200-3.

20. O'Keeffe ST. Secondary causes of restless legs syndrome in older people. Age Ageing 2005; 34: 349-52.

21. O'Keeffe ST. Iron deficiency with normal ferritin levels in restless legs syndrome. Sleep Med 2005; 6: 281-2.

22. Haba-Rubio J, Staner L, Petiau C, Erb G, Schunck T, Macher JP. Restless legs syndrome and low brain iron levels in patients with haemochromatosis. J Neurol Neurosurg Psychiatry 2005; 76: 1009-10.

\section{Restless legs syndrome in patients with high serum ferritin and normal iron levels}

Aim. To document the association between restless legs syndrome (RLS) and high ferritin levels in five patients.

Patients and methods. The five patients were male, mean age: 59 years (range: 36-73 years). The patients were referred for RLS (two of them blood donors), in two cases associated with obstructive sleep apnea. Patients underwent a video-PSC recording. Serum iron and serum ferritin were determined.

Results. All patients fulfilled the clinical criteria for RLS: leg paresthesias associated with an urge to move, motor restlessness, worsening of symptoms during the evening and night, and partial relief with activity, difficulty falling asleep, and presence of nocturnal awakenings due to RLS. Neurological examination, EEGs, EMGs and MRIs were normal. Video-PSGs recordings showed a disturbed and fragmented sleep with a reduction in total sleep time, low sleep efficiency, respiratory abnormalities with an apnea-hipopnea index $>10 / \mathrm{h}$ in two cases, and in all of them a periodic leg movements index $>5 / h$. The serum iron levels were within the normal range in all cases, whereas those in serum ferritin levels were high.

Conclusions. To our knowledge the association of normal serum iron with high serum ferritin levels in patients diagnosed clinically and polygraphically as having RLS with periodic leg movements has not been described before. The notion of an involvement of a dopaminergic mechanism in the pathophysiology of RLS is supported by the decrease in the values of serum ferritin concentration observed in one patient during follow-up while being treated with dopaminergic agents.

Key words. High serum ferritin. Normal iron. Periodic leg movements. Restless legs syndrome. Videopolisomnography. 